\title{
Dinámica a mediano plazo de la revegetación natural de comunidades forestales del centro de Córdoba (Argentina)
}

Karlin, M. S., Cora, A., Bernasconi Salazar, J. R. y Arnulphi, S.

DOI: 10.31047/1668.298x.v37.n1.28068

\begin{abstract}
RESUMEN
Para evaluar la revegetación natural como estrategia de restauración debe comprenderse la dinámica, estructura y regeneración de las comunidades vegetales. El objetivo de este trabajo es analizar los cambios en la estructura forestal de comunidades vegetales del centro de Córdoba, su dinámica y regeneración en el lapso de seis años, evaluando el efecto del ganado y las precipitaciones. En 16 sitios de la Reserva Natural de la Defensa La Calera se relevaron especies forestales y su regeneración $(2013,2016,2019)$ en cuatro comunidades vegetales diferentes. Se excluyó el ganado vacuno de siete sitios y en los nueve restantes estuvo presente en bajas cargas $\left(\sim 12\right.$ ha EV $\left.^{-1}\right)$. Se calcularon los índices de precipitación estandarizados locales. La exclusión del ganado no se tradujo en mayor regeneración. En las comunidades se observó una evolución positiva hacia la madurez, siendo las más dinámicas las de Sebastiania. En las áreas deforestadas, Geoffroea decorticans y Schinus fasciculatus presentan una importante tasa de sobrevida y velocidad de crecimiento. En comunidades de Vachellia y Schinopsis la regeneración sigue la dinámica de las precipitaciones. De acuerdo a los resultados, la revegetación pasiva es una estrategia importante de recuperación en ambientes naturales y semi-naturales del centro de Córdoba.
\end{abstract}

Palabras clave: áreas deforestadas; carga animal; clausura; restauración; Sebastiania; Schinopsis; Vachellia.

Karlin, M. S., Cora, A., Bernasconi Salazar, J. R. and Arnulphi, S. 2020. Mid-term dynamics of the natural revegetation of forest communities in the center of Córdoba (Argentina). Agriscientia 37: 1-13

\section{SUMMARY}

To evaluate the natural revegetation as a restoration strategy, the dynamics, structure and regeneration of the plant communities should be understood. The objective of this work is to analyze the changes in the forest structure of the plant communities in the center of Córdoba, its dynamics, and regeneration along a time lapse of six years, evaluating the effect of cattle and precipitations. In 16 sites located in the Natural Reserve of the Defensa La Calera the woody 
species and their regeneration were surveyed $(2013,2016,2019)$ in four different plant communities. Cattle was excluded from seven sites and it was present in low loads in the other nine $\left(\sim 12\right.$ ha $\left.\mathrm{EV}^{-1}\right)$. The local standardized precipitation indexes were calculated. The cattle exclusion did not lead to higher regeneration. In the communities a positive evolution towards maturity was observed, with the most dynamic communities being those of Sebastiania. In the deforested areas, Geoffroea decorticans and Schinus fasciculatus present an important survival rate and growth speed. In Vachellia and Schinopsis communities the regeneration follows the precipitation dynamics. According to the results, passive revegetation is an important recovery strategy in natural and semi-natural environments of the center of Córdoba.

Key words: deforested areas; livestock load; closure; restoration; Sebastiania; Schinopsis; Vachellia.

Karlin, M. S. (ORCID: 0000-0002-8642-4677) y Arnulphi, S. (ORCID: 0000-0003-3937-5486): Facultad de Ciencias Agropecuarias, Universidad Nacional de Córdoba. Félix Marrone 746, Ciudad Universitaria, Córdoba, Argentina, CP 5000. Asociación Civil El Cuenco - Equipo Ambiental. Manuel Abad e Illana 2337, CP 5000. Córdoba, Argentina. Cora, A. (ORCID: 0000-0003-0530-2422): Asociación Civil El Cuenco - Equipo Ambiental. Manuel Abad e Illana 2337, CP 5000. Córdoba, Argentina. Instituto Nacional de Tecnología Agropecuaria, Estación Experimental Agropecuaria Manfredi, Ruta Nacional № 9, km 636. CP 5986. Manfredi, Córdoba, Argentina. Bernasconi Salazar, J. R. (ORCID: 0000-0001-5619-7242): Asociación Civil El Cuenco - Equipo Ambiental. Manuel Abad e Illana 2337, CP 5000. Córdoba, Argentina. Correspondencia a:mkarlin@agro.unc.edu.ar

\section{INTRODUCCIÓN}

Comprender el funcionamiento de los bosques nativos es fundamental para definir cuáles son las mejores estrategias de restauración. Los programas de restauración con plantación de especies nativas tienen como premisa que estas prácticas aceleran los procesos ecológicos en relación a la revegetación natural. Sin embargo, las tasas de revegetación natural no siempre son menores, y dependerán del grado inicial de disturbio, la disponibilidad de propágulos, las condiciones de suelo y del clima. Además, la revegetación natural suele ser de menor costo (Chazdon, 2008).

Los bosques del Distrito Chaqueño Serrano y de la Provincia Fitogeográfica del Espinal en Argentina (Cabrera, 1976) han sufrido graves procesos de degradación en los últimos 50 años. La composición florística de estos bosques cambia con las características geológicas y edáficas (Cantero et al., 2011; Karlin, Ontibero, Arnulphi y Bernasconi Salazar, 2018), topográficas (Giorgis et al., 2011b) y la historia de disturbio (Brun, Campanella, Oggero y Suárez, 2012; Giorgis, Cingolani, y Cabido, 2013; Bernasconi et al., 2015). Esto determina que el ecosistema sea heterogéneo y que haya variaciones importantes en la composición de especies en toda su extensión (Giorgis et al., 2011a) y en su dinámica de recuperación (Bernasconi et al., 2015).

Las Sierras Chicas de Córdoba y áreas pedemontanas, por su cercanía a centros urbanos, a áreas agrícolas y por su potencial minero y ganadero, experimentan en la actualidad transformaciones causadas por el avance inmobiliario, la minería, la agricultura, los incendios, la tala, el sobrepastoreo y la invasión de especies exóticas. Estas transformaciones condicionan el estado de conservación de los ambientes naturales (Karlin et al., 2014b).

En particular la ganadería es un factor antrópico que, dependiendo de la intensidad y la frecuencia de pastoreo y ramoneo, puede alterar diferentes componentes del ambiente. Conocer el efecto de la ganadería en estos ambientes, bajo condiciones de carga variables, puede dar luz al momento de la aplicación de estrategias de manejo y políticas de regulación.

El éxito de las estrategias de restauración en estos ambientes serranos dependen de múltiples factores entre los que se cuentan el sitio específico 
(Torres et al., 2015), época (Verzino et al., 2004), intensidad de disturbio (Karlin, Arnulphi, Alday, Bernasconi y Accietto, 2016), aplicación de prácticas culturales (Karlin et al., 2014a; Landi y Renison, 2010), características de la vegetación (Karlin et al., 2016), entre otros.

Para evaluar el funcionamiento de dichas estrategias, se necesita primero comprender la dinámica natural, estructura y regeneración de las comunidades vegetales en su hábitat natural. El conocimiento de las tasas de regeneración y de crecimiento de las comunidades puede definir parámetros para evaluar la conveniencia de aplicar estrategias para la revegetación natural o prácticas activas de reforestación.

La Reserva Natural Militar de la Defensa La Calera (RNDLC), ubicada en las laderas orientales de las Sierras Chicas, ha mantenido gran parte de su estructura vegetal original debido a restricciones al ingreso por uso militar. Aquí también se encuentran sectores que han sido disturbados por desmonte y agricultura en el pasado, pero que en su actualidad se encuentran bajo procesos de restauración. Estas situaciones definen un contexto único para evaluar los cambios en la vegetación en el mediano plazo.

La hipótesis del trabajo es que la regeneración y dinámica de las comunidades forestales en las Sierras Chicas y áreas pedemontanas dependen del tipo de vegetación, del ambiente y el clima.
El pastoreo, aunque sea de baja intensidad, es un disturbio que afecta de forma significativa la regeneración de las especies forestales en estos ambientes.

Los objetivos de este trabajo son analizar los cambios temporales en la estructura forestal de formaciones boscosas características de las Sierras Chicas de Córdoba y áreas pedemontanas, su dinámica y regeneración en el lapso de seis años, y evaluar el efecto de las precipitaciones y del pastoreo de baja intensidad sobre la regeneración del sistema.

\section{MATERIALES Y MÉTODOS}

\section{Área de estudio}

Los estudios fueron realizados en la RNDLC, ubicada al oeste de la ciudad de Córdoba. Posee como límites físicos la Ruta Nacional N. ${ }^{\circ} 20$ hacia el sur, la Ruta Provincial N. ${ }^{\circ} 73$ hacia el oeste y el noroeste, el ejido municipal de la localidad de La Calera hacia el norte y parte del anillo de circunvalación de la ciudad de Córdoba hacia el este (Figura 1). Está conformada por tres ambientes geomorfológicos. Por un lado, la Sierra Chica, ambiente serrano ubicado al oeste de la Reserva, caracterizado por bosques de Vachellia spp. y Schinopsis lorentzii (Griseb.) Engl. (=Schinopsis marginata Engl.) en las laderas, y bosques de

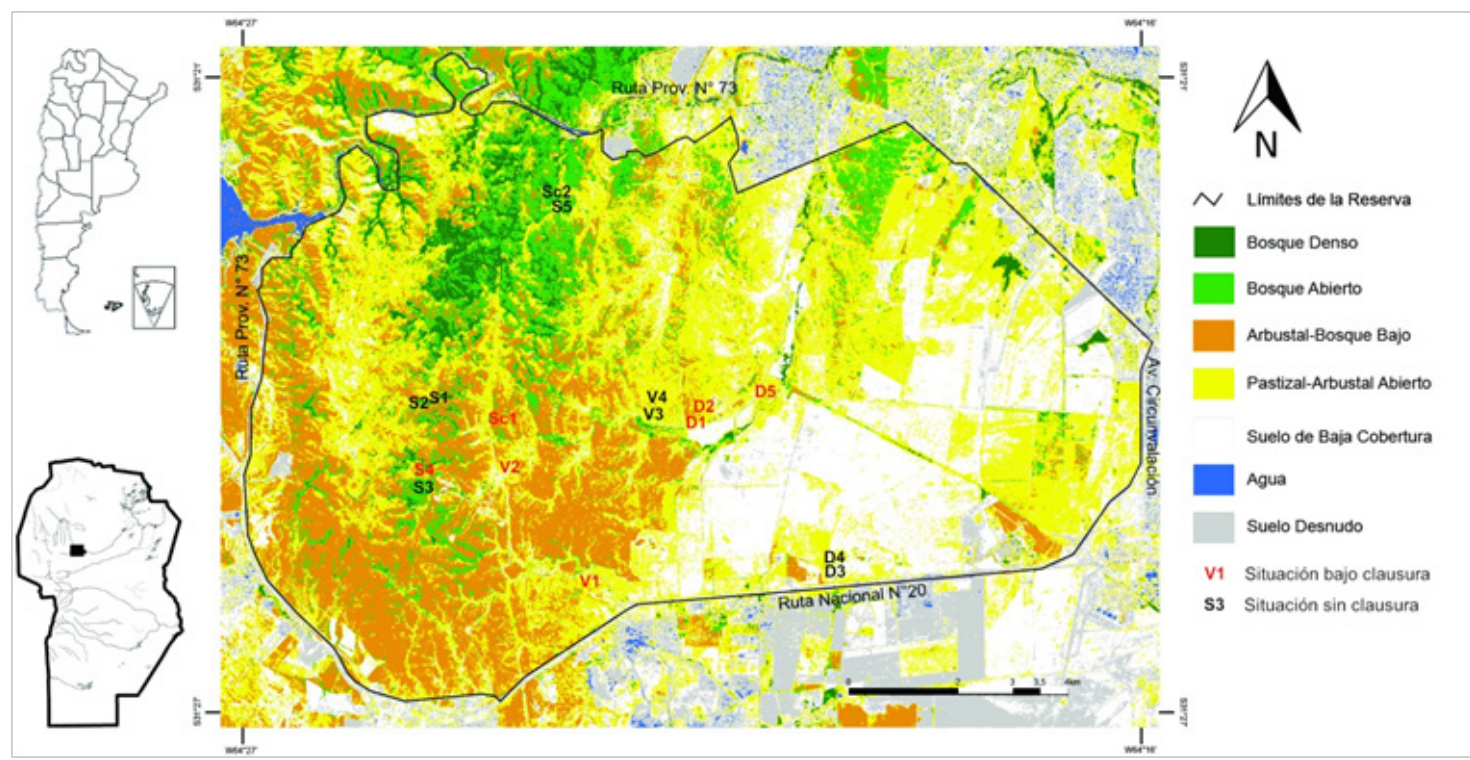

Figura 1. Mapa de ubicación de los sitios de muestreo. (Modificado de Karlin et al., 2014b). V1, V2, V3 y V4: Comunidades de Vachellia spp. S1, S2, S3, S4 y S5: Comunidades de Sebastiania commersionana. Sc1 y Sc2: Comunidades de Schinopsis lorentzii. D1, D2, D3, D4 y D5: Áreas deforestadas. Letras en rojo indican los sitios con exclusión de ganadería. 
Sebastiania commersioniana (Baill.) L. B. Sm. et R. J. Downs en los valles. Por otro lado, la Depresión Periférica, ambiente deprimido al $\mathrm{E}$ del anterior; y el ambiente de la Pampa Loessica Alta, plano elevado respecto al anterior y con pendiente al E; (Karlin et al., 2018). Estos dos últimos representan un área ecotonal entre la vegetación del Distrito del Chaco Serrano y la ecorregión del Espinal (Karlin et al., 2014b), alterados por desmonte para agricultura o pastoreo previamente a ser declarados áreas protegidas en el año 2009, y con escasos parches boscosos de superficie reducida de Aspidosperma quebracho-blanco Schltdl. o Geoffroea decorticans (Gillies ex Hook. \& Arn.) Burkart.

\section{Diseño experimental}

Se identificaron 16 sitios donde se relevaron especies forestales y regeneración, los cuales representan cuatro stands diferentes de especies forestales. Los sitios seleccionados comprenden las siguientes formaciones vegetales:

- Comunidades de Vachellia spp. $(V ; n=4)$, ubicadas en la Sierra Chica, dominados por Vachellia aroma (Gillies ex Hook. \& Arn.) Seigler \& Ebinger y $V$. caven (Molina) Seigler \& Ebinger., asociados a pastizales naturales de gran abundancia que le confieren un elevado riesgo de incendios. Representan el $41 \%$ del área de la RNDLC (Figura 1; Arbustal-Bosque Bajo y parte del Pastizal-Arbustal abierto).

- Comunidades de Sebastiania commersionana (S; $n=5)$, ubicadas en la Sierra Chica, se desarrollan sobre cursos permanentes 0 temporarios de agua. Estas comunidades poseen especies acompañantes como Celtis tala Gillies ex Planch. Se asocian a otras especies exóticas invasoras como el cafeto (Manihot grahamii Hook.) la mora (Morus alba L.) o el siempre verde (Ligustrum lucidum W.T. Aiton). Representa aproximadamente un $10 \%$ de la superficie de la RNDLC (Figura 1; Bosque Denso).

- Comunidades de Schinopsis lorentzii (Sc; $\mathrm{n}=2)$, ubicadas en la Sierra Chica, se desarrollan en áreas de pendientes superiores a $20 \%$ y, al igual que las comunidades de Vachellia spp. están asociados a pastizales naturales de gran abundancia que le confieren un elevado riesgo de incendios. Acompañados por Senegalia praecox (Griseb.) Seigler \& Ebinger como especie secundaria. Representan el $11 \%$ de la superficie de la RNDLC (Figura 1; Bosque Abierto).
- Áreas deforestadas, ubicadas en la Depresión Periférica y Pampa Loéssica Alta, resultantes de desmontes totales para agricultura o pastoreo $(D ; n=5)$. Representan aproximadamente el $35 \%$ del área de la Reserva (Figura 1; Suelo de Baja Cobertura y parte del Pastizal-Arbustal abierto). Si bien no hay especies forestales dominantes, se destacan Geoffroea decorticans y Schinus fasciculatus (Griseb.) I.M. Johnst. como especies pioneras.

Originalmente, en nueve de estos sitios se realizaron clausuras perimetrales de aproximadamente 1 ha $(100 \times 100 \mathrm{~m})$ : V1 y V2; S1, S2 y S4; Sc1; D1, D2 y D5. En los sitios S1 y S2, por motivos ajenos a la planificación del trabajo, ingresaron animales que provocaron efectos de sobrepastoreo, por lo que se les cambió la categoría a "no clausura". La clausura consistió en alambrado perimetral de cinco hilos, excluyendo desde mayo del año 2013 hasta las mediciones de 2019 el ganado vacuno presente en la Reserva. Si bien las clausuras se plantearon como tratamiento de exclusión del ganado, debe tenerse en cuenta que las cargas animales dentro de la Reserva son y han sido siempre reducidas. Se estima que las cargas son de aproximadamente 500 animales sobre un área de pastoreo mínima de 6000 ha $\left(\sim 12\right.$ ha $\left.E^{-1}\right)$, considerando que la receptividad estimada en el área serrana de la Reserva, según datos de biomasa herbácea de Karlin et al. (2015) y aplicando un factor de uso de 0,5, es de 5,48 ha $\mathrm{EV}^{-1}$.

\section{Mediciones forestales}

Para las mediciones forestales se instalaron transectas en faja fijas (Gaillard de Benitez y Pece, 2011), identificadas con estacas, cuyas dimensiones fueron de $3 \times 50 \mathrm{~m}$. En cada una se midió el número de árboles (nativos y exóticos invasores), diámetro a la altura de la base $(D A B)$ de cada uno y cantidad de renovales, considerando como tal aquellos individuos de menos de $5 \mathrm{~cm}$ de $D A B$ y de cualquier altura.

Para la medición de los stands forestales se definieron dos fechas, 2013 (línea de base) y 2019, mientras que para renovales se hizo además una medición intermedia en 2016. Las mediciones se efectuaron entre junio y octubre de forma de comparar épocas similares.

\section{Datos pluviométricos}

Se obtuvieron los registros meteorológicos de una estación automática ubicada a 400 m 
al suroeste de V2 (Figura 1). De la estación pudieron obtenerse los datos correspondientes a los períodos noviembre de 2012 hasta febrero de 2015 y datos aislados de 2016. Los baches de información se deben a un mal funcionamiento de la estación.

En paralelo se obtuvieron datos pluviométricos del Aeropuerto Pajas Blancas de Córdoba (CBA AERO) ubicado a $20 \mathrm{~km}$ del área de estudio, a fin de contrastar los datos propios y para estimar y/o ajustar los faltantes.

Dichos datos se condensaron para obtener índices anuales de pluviometría, los cuales fueron analizados para los períodos julio-junio en cada año. Las estimaciones se realizaron a través de un modelo lineal de correlación entre los datos de CBA AERO y los disponibles de la estación automática.

Con los datos ajustados de precipitación se calcularon los índices de precipitación estandarizados (SPI en inglés) según la metodología de cálculo explicada en Naresh Kumar, Murthy, Sesha Sai y Roy (2009). Estos datos fueron graficados a fin de analizar los ciclos húmedos y secos entre mediciones.

\section{Análisis estadístico}

Los datos de las transectas se extrapolaron a valores por hectárea, definiendo así el número de individuos adultos (Ind) y renovales (Ren). Con estas dos variables se estableció el índice Ind/Ren como relación de la cantidad de árboles semilleros y la producción de renovales. Para cada sitio y población se calculó el área basal $(A B)$ de los stands forestales y el área basal individual $\left(A B_{\text {ind }}\right)$ tomando como base el DAB. Se discriminó el área basal de las especies exóticas forestales invasoras, calculando el porcentaje de $A B$ sobre el total $\left(\% A B_{e}\right)$.

Se confeccionaron diagramas de clase diamétrica por comunidad vegetal tomando los valores promedio de cada una para cada año, considerando en cada comunidad las dos especies forestales más abundantes y agrupando el resto en las categorías "Otras Nativas" y "Exóticas". Se graficaron diagramas de barra para densidad de renovales en las tres fechas.

Puesto que los datos no superaron la prueba de normalidad de Shapiro-Wilks, se realizaron análisis de varianza no paramétricos (KruskalWallis) y análisis discriminante mediante el programa estadístico InfoStat (Di Rienzo et al., 2019). Se compararon los valores de la variable
Ren según tratamiento de clausura / no clausura. Se compararon el total de las variables según año de medición, discriminado por comunidad. Se compararon también las frecuencias de las clases diamétricas, según año. Se aplicaron regresiones lineales entre las variables Ren, Ind y $A B$.

A fin de sintetizar los valores de SPI de los períodos comprendidos entre mediciones, estos fueron ponderados $\left(S P I_{\text {pond }}\right.$ ) de acuerdo a la antigüedad del valor respecto al momento de medición. Es decir, para la obtención del índice se aplicó la siguiente ecuación:

$$
S P I_{\text {pond }}=\left(S P I_{j}^{*} 0,4\right)+\left(S P I_{j-1}{ }^{*} 0,3\right)+\left(S P I_{j-2} * 0,2\right)+\left(S P I_{j-3}{ }^{*} 0,1\right)
$$

donde $S P I$ es el índice de precipitación estandarizado anualizado y $j$ es el año en que se efectuó la medición forestal (2013, 2016 o 2019). De esta forma se considera de forma indirecta el agua almacenada en el suelo y los acuíferos como consecuencia de las precipitaciones de años anteriores.

\section{RESULTADOS Y DISCUSIÓN}

El período de seis años analizado estuvo caracterizado por un régimen cíclico de precipitaciones, tal como se muestra en la Figura 2. Se aprecia inicialmente un período 2010-2013 seco, con un $S P I_{\text {pond }}$ negativo; un período 20132016 húmedo, con un $S P I_{\text {pond }}$ positivo, y finalmente un período 2016-2019 con un período bianual seco, recuperándose en el último año, obteniendo un $S P l_{\text {pond }}$ indiferente.

La comparación entre tratamientos (clausurado / no clausurado), particionado por año (2013, 2016 y 2019) no arrojó diferencias estadísticamente significativas en el número de renovales por hectárea, considerada la variable más sensible a ocasional ramoneo (2013: clausura $=3514$, no clausura=3269 Ren ha-1; $H=1,89 ; \quad p=0,183$; 2016: clausura $=5513$, no clausura $=5538$ Ren ha $^{-1} ; \quad H=0,34 ; p=0,606 ;$ 2019: clausura $=4833$, no clausura=9010 Ren ha-1; $H=0,47 ; p=0,536)$. A pesar de la presencia de ganado en la RNDLC y la reconocida apetecibilidad por los renovales de especies forestales (Karlin et al., 2015; Barberá, Renison y Torres, 2018), las cargas ganaderas reducidas no ejercieron mayor influencia sobre la regeneración, por lo que se rechaza parte de la hipótesis inicial. Es notable la diferencia de medias en 2019 a favor del tratamiento "no clausura"; a pesar de esto, no resulta ser significante debido a la gran amplitud de valores obtenidos en este

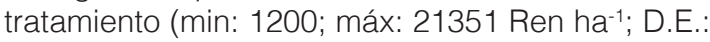
8435,81; CV: 93,63). 


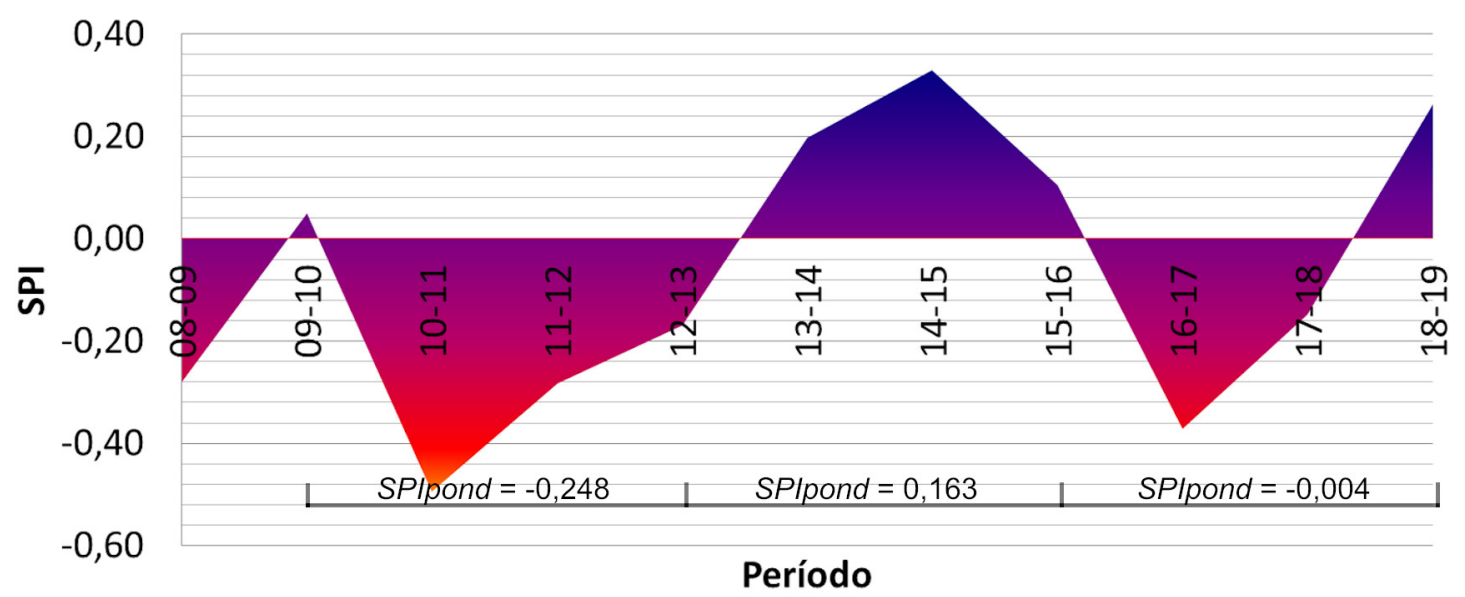

Figura 2. Diagrama temporal del Índice Estandarizado de Precipitaciones (SPI) para el área de estudio (azul, más húmedo; rojo, más seco).

Es posible que a pesar de la apetecibilidad por los renuevos, una mayor disponibilidad de biomasa herbácea por efecto de bajas cargas ganaderas, reduzca la presión de ramoneo de renuevos. Esto es importante ya que la presencia de ganado vacuno en bajas cargas podría colaborar en la reducción de la biomasa fácilmente combustible, reduciendo el riesgo de incendios (Karlin et al., 2015), sin comprometer la regeneración del bosque, aunque se debe monitorear el impacto del pisoteo sobre el suelo ya que se ha comprobado que la capacidad de infiltración de los suelos se reduce de manera importante (Karlin et al., 2019).

Los resultados de los relevamientos sobre las comunidades de Vachellia spp., S. commersioniana, S. lorentzii y áreas deforestadas seleccionadas se muestran en la Tabla 1. Se observan incrementos variables en la cantidad de individuos, renovales y área basal en 2019 respecto a 2013.

En la Figura 3 se grafican las distribuciones diamétricas por comunidad. En todas las comunidades y en ambas fechas se observaron curvas de distribución diamétrica en forma de "J" invertida.

El área basal se incrementa, como era esperado, en todas las comunidades y se relaciona al aumento de individuos por hectárea mediante la relación $A B=-0,75+0,01^{*} /$ nd $(F=68,77 ; p<0,0001$; CpMallows $\left.=68,77 ; R^{2}=0,70\right)$.

Las comunidades de Vachellia (V) son, con excepción de las áreas deforestadas, las de menores cantidades de individuos por hectárea y menor $A B$, debido a que están dominadas por especies del género Vachellia, las cuales rara vez alcanzan clases diamétricas grandes (Tabla 1). El

Tabla 1. Resumen del número por hectárea de individuos adultos (Ind), renovales (Ren), relación Ind/Ren, área basal $(A B)$ y área basal individual ( $A B_{\text {ind }}$ ), para 2013 y 2019 (y 2016 en Ren). E.E.: Error estándar. V: comunidades de Vachellia spp.; S: comunidades de Sebastiania commersioniana; Sc: comunidades de Schinopsis lorentzii; D: áreas deforestadas; $\Delta \%$ : porcentaje de cambio entre 2019 y 2013; $p$ : $p$-valor del test Kruskal-Wallis. Letras diferentes indican diferencias estadísticamente significativas $(=0,05)$.

\begin{tabular}{|c|c|c|c|c|c|c|c|c|c|c|c|c|c|c|c|c|c|c|c|}
\hline & \multicolumn{4}{|c|}{ Ind $\left(\mathrm{ha}^{-1}\right)$ (E.E.) } & \multicolumn{5}{|c|}{$\operatorname{Ren}\left(\mathrm{ha}^{-1}\right)$ (E.E.) } & \multicolumn{2}{|c|}{$\begin{array}{c}\text { Ind/Ren } \\
\left(\mathrm{E}_{\mathrm{E}} \mathrm{E}_{\mathrm{S}}\right)\end{array}$} & \multicolumn{4}{|c|}{$A B\left(m^{2} h^{-1}\right)$ (E.E.) } & \multicolumn{4}{|c|}{$A B_{\text {ind }}\left(\mathrm{m}^{2}\right.$ ind $\left.^{-1}\right)(\mathrm{E} . \mathrm{E})}$. \\
\hline & 2013 & 2019 & $\%$ & $p$ & 2013 & 2016 & 2019 & $\Delta \%$ & $p$ & 2013 & 2019 & 2013 & 2019 & $\Delta \%$ & $p$ & 2013 & 2019 & $\Delta \%$ & $p$ \\
\hline$n=4)$ & $\begin{array}{c}467 \\
(103)\end{array}$ & $\begin{array}{c}688 \\
(134)\end{array}$ & 47,3 & 0,343 & $\begin{array}{c}1950 \mathbf{a} \\
(91)\end{array}$ & $\begin{array}{c}3850 \mathbf{b} \\
(917)\end{array}$ & $\begin{array}{c}3563 \mathbf{b} \\
(750)\end{array}$ & 82,7 & & $5 \begin{array}{c}0,24 \\
(0,05)\end{array}$ & $\begin{array}{c}0,20 \\
(0,05)\end{array}$ & $\begin{array}{c}2,56 \\
(0,39)\end{array}$ & $\begin{array}{c}4,04 \\
(1,56)\end{array}$ & & 0,886 & $\begin{array}{l}5,8 \cdot 10^{-4} \\
\left(4.10^{-5}\right)\end{array}$ & $\begin{array}{l}6,0.10^{-4} \\
\left(2.10^{-4}\right)\end{array}$ & 3,45 & 0,999 \\
\hline ( & $\begin{array}{l}1629 \\
(324)\end{array}$ & $\begin{array}{l}2094 \\
(418)\end{array}$ & 28,5 & 0,548 & $\begin{array}{c}5000 \\
(1620)\end{array}$ & $\begin{array}{c}8676 \\
(3369)\end{array}$ & $\begin{array}{l}13094 \\
(4232)\end{array}$ & 161 & 0,527 & $\begin{array}{c}0,43 \\
(0,13)\end{array}$ & $\begin{array}{c}0,43 \\
(0,21)\end{array}$ & $\begin{array}{l}19,50 \\
(4,70)\end{array}$ & $\begin{array}{l}28,01 \\
(7,27)\end{array}$ & 43,6 & 0,548 & $\begin{array}{l}1,3 \cdot 10^{-3} \\
\left(4.10^{-4}\right)\end{array}$ & $\begin{array}{l}1,4 \cdot 10^{-3} \\
\left(3.10^{-4}\right)\end{array}$ & 11,1 & 0,841 \\
\hline 2) & $\begin{array}{c}633 \\
(167)\end{array}$ & $\begin{array}{l}975 \\
(25)\end{array}$ & 54,0 & 0,333 & $\begin{array}{c}3133 \\
(1267)\end{array}$ & $\begin{array}{c}6483 \\
(3883)\end{array}$ & $\begin{array}{l}5725 \\
(2175)\end{array}$ & 82,7 & 0,800 & $\begin{array}{c}0,22 \\
(0,03)\end{array}$ & $\begin{array}{c}0,20 \\
(0,08)\end{array}$ & $\begin{array}{c}6,72 \\
(1,85)\end{array}$ & $\begin{array}{c}9,56 \\
(1,28)\end{array}$ & 3 & 0,667 & $\begin{array}{l}1,1.10^{-3} \\
\left(1.10^{-5}\right)\end{array}$ & $\begin{array}{l}9,8.10^{-4} \\
\left(1.10^{-4}\right)\end{array}$ & -7, & 0,999 \\
\hline$(n=5)$ & $\begin{array}{c}60 \\
(40)\end{array}$ & $\begin{array}{l}143 \\
(34)\end{array}$ & 138,3 & 0,191 & $\begin{array}{l}2990 \\
(733)\end{array}$ & $\begin{array}{l}3339 \\
(536)\end{array}$ & $\begin{array}{c}4750 \\
(1846)\end{array}$ & 58,9 & & $2 \begin{array}{c}0,02 \\
(0,01)\end{array}$ & $\begin{array}{c}0,05 \\
(0,01)\end{array}$ & $\begin{array}{c}0,84 \\
(0,93)\end{array}$ & $\begin{array}{c}1,09 \\
(0,37)\end{array}$ & 29,8 & 0,413 & $\begin{array}{l}6,2.10^{-4} \\
\left(5.10^{-4}\right)\end{array}$ & $\begin{array}{l}5,4.10^{-4} \\
\left(1.10^{-4}\right)\end{array}$ & $-12,9$ & 0,413 \\
\hline romedio & $\begin{array}{c}724 \\
(193)\end{array}$ & $\begin{array}{c}993 \\
(240)\end{array}$ & 37,2 & 0,327 & $\begin{array}{l}3376 \\
(608)\end{array}$ & $\begin{array}{c}5527 \\
(1224)\end{array}$ & $\begin{array}{c}7183 \\
(1712)\end{array}$ & 112 & 0,183 & & & $\begin{array}{c}7,92 \\
(2,49)\end{array}$ & $\begin{array}{l}11,22 \\
(3,68)\end{array}$ & & 51 & $\begin{array}{l}8,6.10^{-4} \\
\left(2.10^{-4}\right)\end{array}$ & $\begin{array}{l}8,8.10^{-4} \\
\left(2.10^{-4}\right)\end{array}$ & 2,32 & 0,910 \\
\hline
\end{tabular}




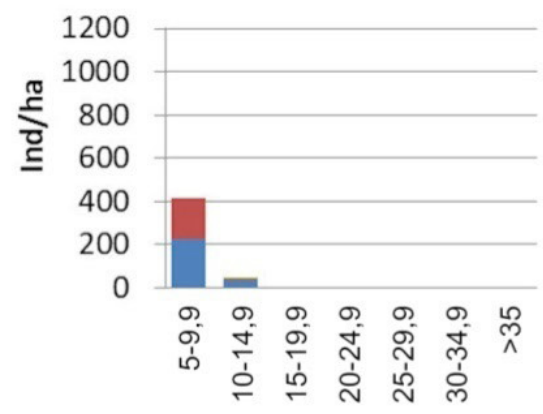

Clases diamétricas $(\mathrm{cm})$

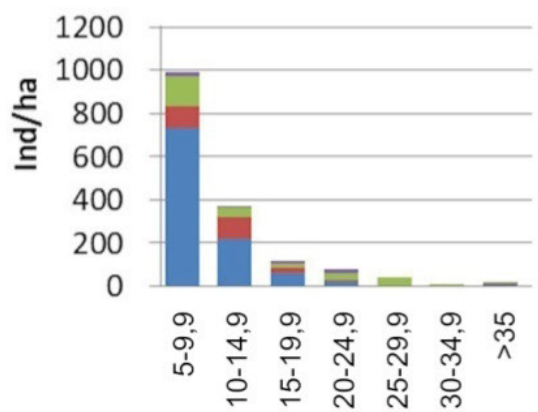

Clases diamétricas $(\mathrm{cm})$

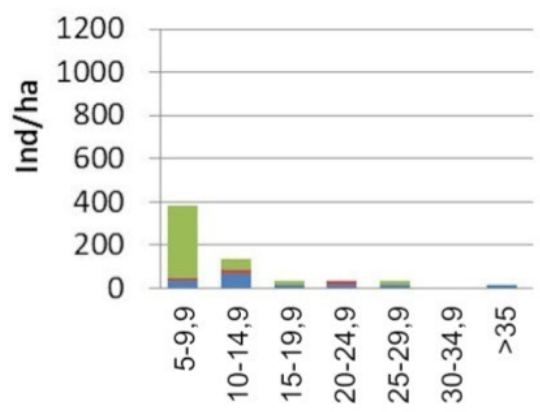

Clases diamétricas $(\mathrm{cm})$

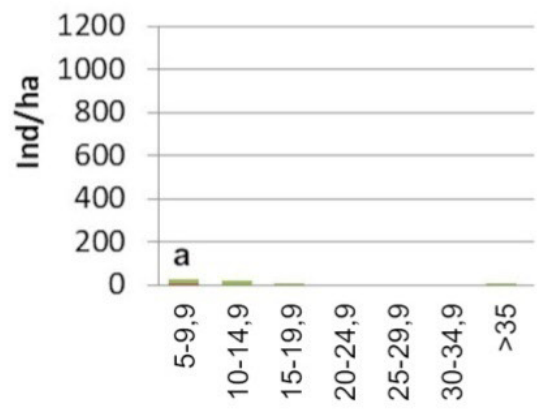

Clases diamétricas $(\mathrm{cm})$

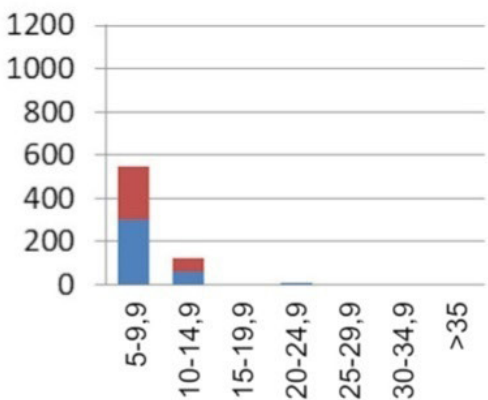

Clases diamétricas $(\mathrm{cm})$

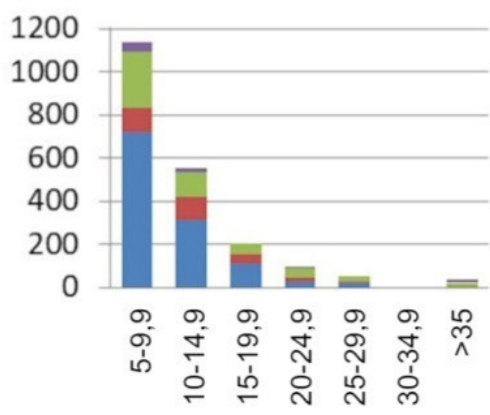

Clases diamétricas $(\mathrm{cm})$

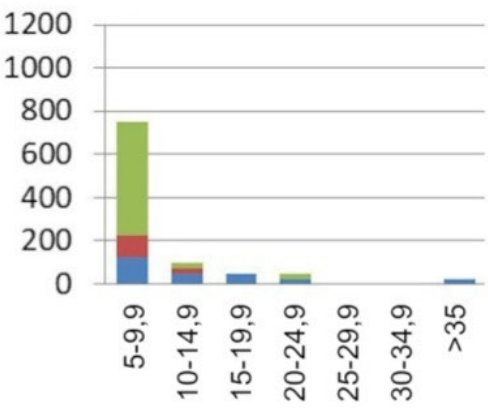

Clases diamétricas $(\mathrm{cm})$

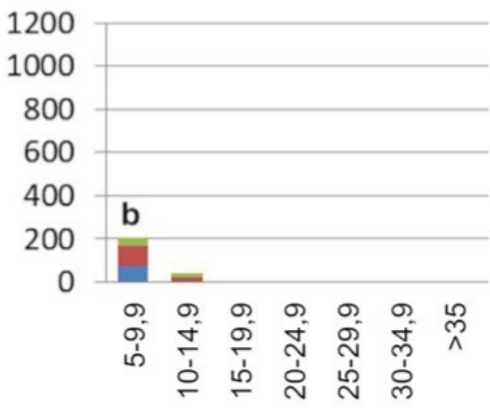

d

- Otras Nativas

S. fasciculatus

G. decorticans

Figura 3. Distribución diamétrica de la estructura arbórea en comunidades de Vachellia spp. (a), S. commersioniana (b), S. Iorentzii (c) y áreas deforestadas (d). Izq.: 2013, der.: 2019. 
número de individuos y área basal en seis años supera en casi un 50 \% la línea de base, mientras que los renovales se incrementan en poco más del $80 \%$ de forma significativa. Estas comunidades son las que poseen menor cantidad de renovales por hectárea y presentan mayor tasa de incremento de $A B$. No se han observado individuos de especies forestales exóticas.

Las comunidades de Sebastiania (S) poseen la mayor densidad de individuos y presentan las mayores tasas incrementales de $A B_{\text {ind }}$. Presentan también las mayores cantidades de renovales y tasas incrementales, aunque la cantidad de renovales por individuo adulto es la menor (es decir, poseen alta relación Ind/Ren) (Tabla 1). El área basal de las especies exóticas forestales invasoras en las comunidades de $S$. commersioniana se redujo $-31,4 \%$ en $2019(8,71$ $\pm 6,96 \mathrm{~m}^{2}$ ha $\left.^{-1}\right)$ respecto a $2013\left(12,70 \pm 6,96 \mathrm{~m}^{2}\right.$ ha $\left.^{-1}\right)$, aunque de manera no significativa $(H=0,04$; $p=0,897)$. Es probable que esta reducción se deba a que algún individuo de gran fuste no haya sido registrado en alguna de las transectas por caída o bien por corrimiento de la cinta de la transecta en 2019 respecto a 2013.

Las comunidades de Schinopsis (Sc) poseen valores intermedios entre los de Vachellia y Sebastiania en el número de individuos y número de renovales por hectárea, aunque se comporta más parecido a los stands de Vachellia cuando se analizan la relación Ind/Ren y las tasas de cambio de Ind y Ren. Presentan una tasa de incremento de $A B$ similar a Sebastiania. Presenta un incremento (no significativo: $H=2,40 ; p=0,333$ ) en la frecuencia de la clase diamétrica 0,05-0,099 m de casi el $100 \%$. La tasa de $A B_{\text {ind }}$ se reduce en los 6 años y no posee especies exóticas. A pesar de que las tasas incrementales de $A B$ en las comunidades de Schinopsis son reducidas, las tasas iniciales de crecimiento son elevadas, evidenciadas en el incremento (aunque no significativo) de la frecuencia en la clase diamétrica 0,05-0,099 m. Este importante incremento puede ser el responsable por el cual las tasas en $A B_{\text {ind }}$ son negativas.

Las áreas deforestadas (D) se caracterizan por una baja relación Ind/Ren debido a la baja cantidad de individuos adultos (< 150 ind ha $\left.{ }^{-1}\right)$. A pesar de ello la cantidad de renovales encontrados es importante como consecuencia de que dichas áreas se ubican en zonas aledañas a parches remanentes de bosque que permiten dispersar propágulos. De hecho, se observa un incremento significativo $(H=4,81 ; p=0,0317)$ en la frecuencia de individuos de la clase diamétrica 0,05 - 0,099 (Figura 3d) entre 2013 y 2019. Las especies G. decorticans y $S$. fasciculatus son las de mayor importancia en términos de colonización de áreas descubiertas; el primero de altas tasas de dispersión y crecimiento debido a la capacidad de dispersarse agámicamente mediante raíces gemíferas (Burkart, 1949); el segundo debido a una elevada tasa de dispersión de semillas y éxito en su germinación y emergencia. La significancia obtenida en la clase diamétrica 0,050,099 m entre 2013 y 2019 indica una importante tasa de sobrevida de los renovales y velocidad de crecimiento de estas especies, por lo que se las podría considerar importantes agentes de restauración de ambientes deforestados. S. fasciculatus y $G$. decorticans son especies resistentes al ramoneo (Capó, Aguilar y Renison, 2016), por lo que sitios que han sido desmontados, utilizados para agricultura y posteriormente abandonados, como en el presente caso, pueden ser rehabilitados aplicando un pastoreo racional que evite una acumulación de biomasa herbácea que pueda incrementar la competencia sobre renuevos forestales o incrementar el riesgo de incendios.

La estructura en forma de "J" invertida de las diferentes comunidades forestales y los incrementos en el número de individuos adultos sugieren una evolución positiva de estos ambientes hacia la madurez. La falta de significancia en las frecuencias obtenidas de las clases diamétricas en el lapso de seis años en las comunidades de Vachellia, Schinopsis y Sebastiania sugieren que los casos evaluados presentan una gran heterogeneidad y debería incrementarse el número de observaciones, o bien alargar el lapso de observaciones.

La cantidad de renovales es la variable de respuesta más rápida en un análisis de mediano plazo como el que se presenta aquí, por ello merece un análisis detallado. Cuando se analiza en términos globales se aprecia en la Tabla 1 que entre 2013 y 2019 ha habido diferencias (aunque no estadísticamente significativas), con incrementos promedio de más del $100 \%$ respecto a la línea de base.

En la Figura 4 se muestran los cambios específicos y cuantitativos de renovales forestales en las comunidades analizadas.

En las comunidades de Vachellia los valores totales de renuevos se incrementan a casi el doble entre 2013 y 2016, con una ligera baja en 2019, siguiendo la tendencia de las precipitaciones. Ambas especies de Vachellia (aroma y caven) incrementan su cantidad respecto a la línea base.

En las comunidades de Sebastiania los incrementos del total de renovales son 

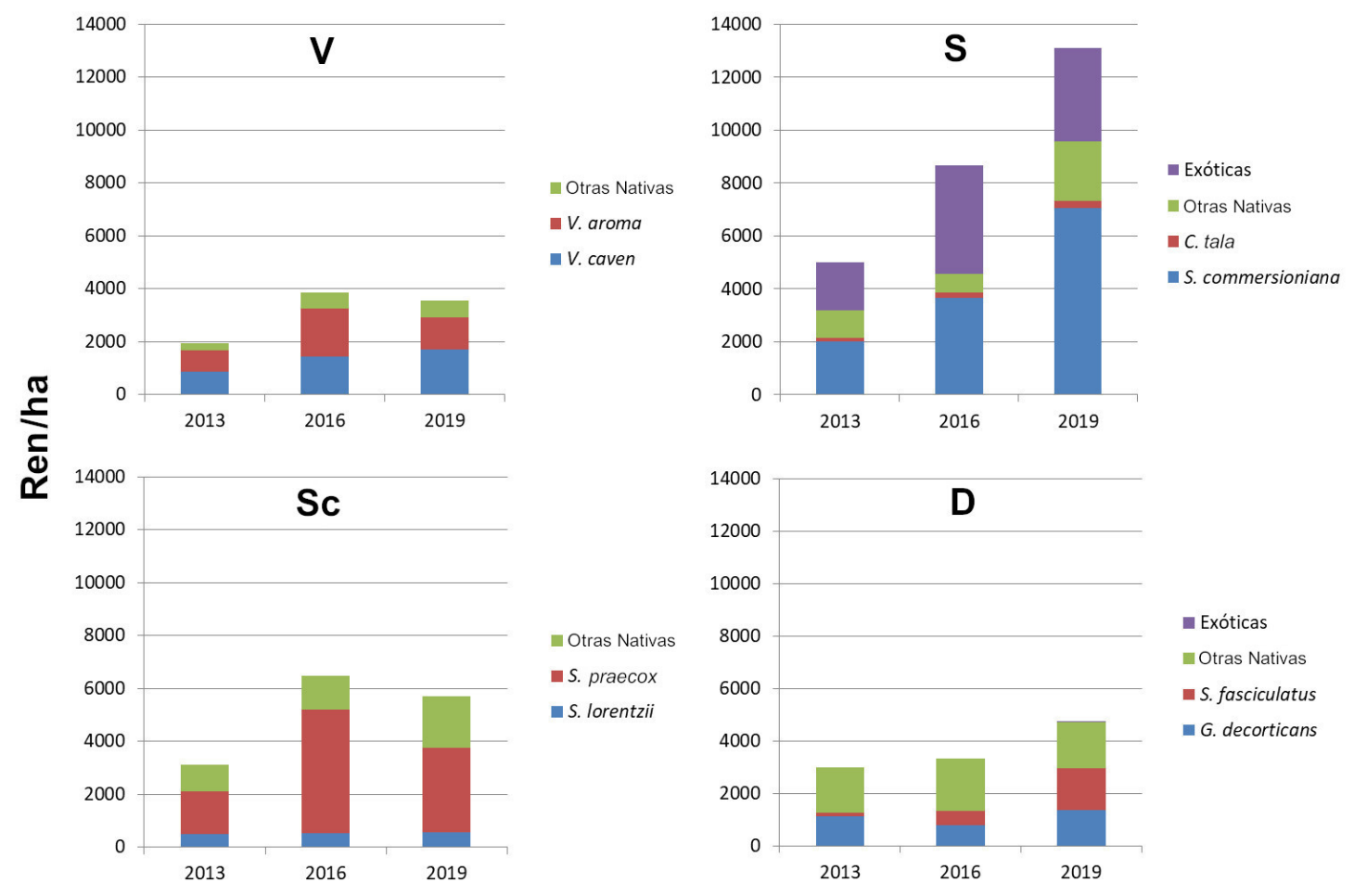

\section{Año}

Figura 4. Renovales por hectárea por comunidad analizada y por año. V: Comunidad de Vachellia spp.; S: comunidad de Sebastiania commersioniana; Sc: comunidad de Schinopsis lorentzii; D: áreas deforestadas.

proporcionales, aunque los de $C$. tala se mantienen relativamente constantes entre 2016 y 2019 (227 y 285 Ren ha ${ }^{-1}$, respectivamente vs 127 Ren ha $^{-1}$ en 2013). Se observa el incremento de renovales de especies nativas (3180, 4569 y 9567 Ren ha $^{-1}$ en 2013, 2016 y 2019, respectivamente) y también el de exóticas (1820, 4017 y 3528), aunque la relación nativa/exótica disminuye a los tres años para luego aumentar en 2019 (1,75, 1,13 y 2,71).

En las comunidades de Schinopsis el comportamiento de los renovales es similar a las comunidades de Vachellia. Si bien hay un ligero incremento en los renovales de S. lorentzii (483, 525 y 575 Ren ha-1 para 2013, 2016 y 2019, respectivamente), los renovales de $S$. praecox (1633, 4675 y 3175) son los dominantes. Sin embargo, muy pocos individuos de esta especie alcanzan la madurez.

En las áreas deforestadas, G. decorticans mantiene su número de renovales relativamente estable en el tiempo (1130, 815 y 1363 Ren ha $^{-1}$ para 2013, 2016 y 2019, respectivamente), mientras que se incrementa de forma importante la frecuencia de $S$. fasciculatus (140, 517 y 1606). Ambas especies son colonizadores tempranos en ambientes deforestados, aportando a incrementar la proporción de individuos de clases diamétricas bajas (Figura 3d) en el término de seis años.

La regeneración tiende en todos los casos a aumentar respecto a la línea de base (2013). Sin embargo, las comunidades de Vachellia y Schinopsis, muy similares en cuanto a estructura, riqueza y diversidad (Karlin et al., 2014b), sin influencia de vías de escurrimiento presentes en el área de estudio, siguen un patrón asociado a los ciclos de precipitación; en ciclos secos la regeneración se reduce, y viceversa, mientras que con SPI ${ }_{\text {pond }}$ indiferentes (ciclo 2016-2019) la regeneración es intermedia. Esta relación debe ser estudiada en mayor detalle en el futuro.

En la Figura 5 se muestra la síntesis multivariada de los cambios en cada una de las comunidades.

En este análisis se discriminan las cuatro comunidades, separando los casos según los años 2013 y 2019. Pueden así identificarse las trayectorias temporales en dicha Figura. Puede 


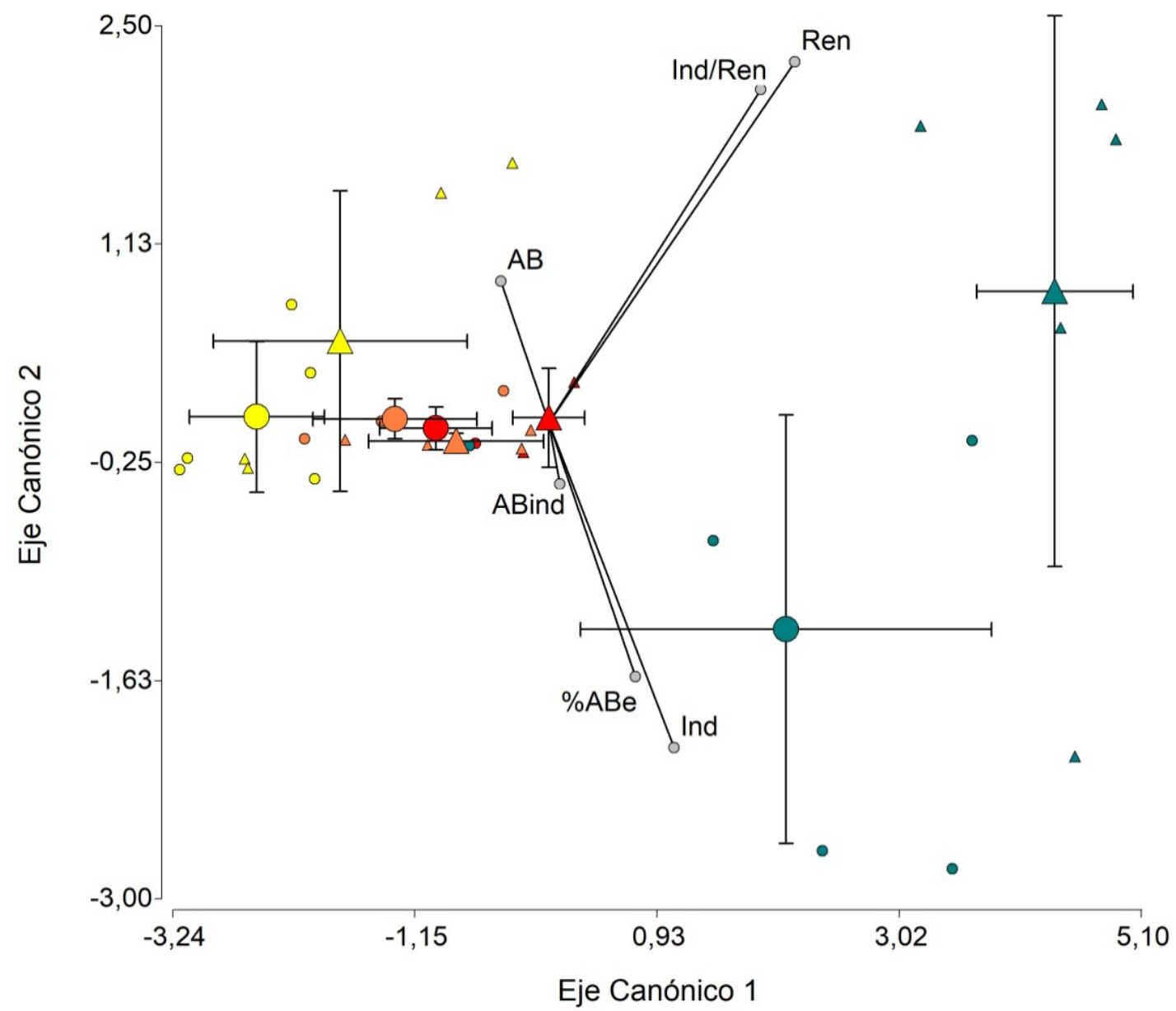

Figura 5. Biplot de análisis discriminante por comunidad y año (2013 y 2019) en función a las variables analizadas. Símbolos pequeños corresponden a cada uno de los casos, símbolos grandes corresponden a los centroides con las desviaciones estándar. Ind: cantidad de individuos por hectárea; Ren: cantidad de renovales por hectárea; Ind/Ren: relación cantidad de individuos adultos y renovales; $\% A B_{e}$ : porcentaje del área basal de especies exóticas; $A B$ : área basal de los stands forestales; $A B_{\text {ind }}$ área basal individual. Círculos: 2013; triángulos: 2019. Verde: comunidades de Sebastiania; rojo: comunidades de Schinopsis; anaranjado: comunidades de Vachellia; amarillo: áreas deforestadas.

observarse un agrupamiento de las comunidades de Sebastiania, por un lado, y el resto (Vachellia, Schinopsis y áreas deforestadas) por el otro.

Las comunidades más dinámicas desde el punto de vista forestal son las de Sebastiania (S). Estas se relacionan positivamente con la variable Ren, incrementándose en el tiempo. El efecto de las precipitaciones (ciclos húmedos y secos) sobre la regeneración puede verse enmascarado quizá porque el agua no es limitante debido a su ubicación en vías de escurrimiento y a que el perfil de suelo se mantiene con humedad durante todo el año. Al contrario, las áreas deforestadas (D) están inversamente relacionados a la variable
Ren, aunque su evolución tiende a un incremento en el número de renovales. Ambos extremos, S y $D$, varían quizá por diferencias en la disponibilidad de árboles semilleros (Ren $=2826,13+2,86^{\star}$ Ind; $\mathrm{p}=0,0085 ;$ CpMallows $\left.=7,94 ; R^{2}=0,18 ; A / C=638,46\right)$ yárea basal $\left(R e n=3616,73+173,77^{\star} A B ; p=0,0230\right.$; CpMallows $=5,74 ; R^{2}=0,13 ; A / C=640,37$ ), variables que dinamizan la regeneración.

En las áreas deforestadas la tasa de regeneración es siempre creciente posiblemente por la reducida competencia interespecífica, e independiente de la pluviometría. La especie que más condiciona esta regeneración es $S$. fasciculatus; esta es una especie promisoria para 
ser utilizada en restauración de áreas degradadas. Natale, Oggero, Marini y Reinoso (2014) determinaron que es una especie que germina con facilidad, es sumamente resistente a la sequía y presenta elevadas tasas de supervivencia. Esta resistencia a la sequía quizá los haga indiferentes a los ciclos de humedad y sequía predominantes. Es probable que la regeneración dependa más de la disponibilidad de luz, factor que no es limitante en estos stands, hasta tanto se alcance un umbral crítico de cobertura que reduzca la luminosidad.

En la Figura 5 también puede observarse que las comunidades de Sebastiania están directamente relacionadas con \%AB ya que sólo en estos sitios se encontraron especies exóticas forestales en cantidades significativas, aunque esta relación es más débil en 2019 por la reducción de los valores de esta variable.

También puede observarse que las comunidades de Vachellia y Schinopsis se superponen y mantienen una trayectoria similar entre 2013 y 2019. Esto sugiere que ambos ambientes podrían ser parte de una misma sucesión ecológica (Bernasconi et al., 2015) o bien que podrían ser manejados de forma semejante (Karlin et al., 2015).

Los incrementos, en términos generales, del número total de individuos, renuevos y área basal son muy variables espacialmente y esto conduce a dificultades en la aplicación de métodos estadísticos para evaluar la evolución de estos ambientes complejos en términos absolutos. Los seis años de evaluación muestran cambios importantes, aunque estas variables deben seguir siendo monitoreadas en el tiempo a fin de darle tiempo al sistema para que magnifique su expresión.

La revegetación natural puede constituir una interesante estrategia de recuperación de ambientes naturales o semi-naturales en algunos sectores de las Sierras de Córdoba, especialmente en sitios donde el agua no constituye una limitante importante, como en áreas riparias, o bien donde la competencia interespecífica es reducida, tal como ocurre en áreas de desmonte. En áreas riparias debe estudiarse en mayor detalle la eficiencia de la revegetación natural de especies nativas en áreas con distintos grados de invasión de exóticas. En áreas deforestadas la presencia de núcleos semilleros sería fundamental para la propagación de semillas y aparición de renovales. En comunidades de Vachellia y Schinopsis, que se desarrollan generalmente en laderas secas de zonas serranas y con suelos poco profundos, esta estrategia de revegetación natural también debe ser contemplada en comparación a acciones de restauración activa tales como plantaciones. Las plantaciones dependen fundamentalmente de la disponibilidad de agua en los primeros estadíos y suelen tener una sobrevida reducida (Verzino et al., 2004), lo que hace de esta estrategia bastante costosa.

\section{CONCLUSIONES}

Los resultados obtenidos de la revegetación natural en las comunidades forestales evaluadas en este trabajo son promisorios y deberían ser profundizados y validados en otros contextos de restauración y rehabilitación pasivas de las Sierras de Córdoba y zonas pedemontanas. Esta estrategia de relativo bajo costo podría ser incluso aplicada en planes de conservación que consideren actividades de pastoreo con bajas cargas.

Promover la regeneración es fundamental para asegurar la consolidación de stands forestales en el futuro. Si bien no se encontró significancia estadística en muchas de las variables analizadas, las tendencias en la regeneración y en el desarrollo de las clases diamétricas fueron hacia el alza y se recomienda ampliar los períodos de evaluación.

En zonas riparias la revegetación puede ser importante gracias a que la disponibilidad de agua no es limitante, sin embargo, debe evaluarse la eficiencia de estas estrategias pasivas en ámbitos donde la invasión de especies exóticas sea mayor a la del área de estudio.

En áreas deforestadas la regeneración natural depende de la presencia de árboles semilleros en zonas aledañas; una mayor cantidad de renuevos está relacionada a mayor cantidad de individuos adultos semilleros y a áreas basales elevadas. Se recomienda evaluar la regeneración en zonas alejadas a núcleos semilleros.

La regeneración en stands de Vachellia y Schinopsis se vería afectada por las precipitaciones y los ciclos de sequía/humedad, aunque este efecto debe ser estudiado en mayor detalle. La ocurrencia de ganadería en bajas cargas dentro de estos ambientes de gran producción de biomasa fácilmente combustible podría ayudar a reducir el riesgo de incendios sin comprometer la regeneración forestal por efecto de ramoneo.

\section{AGRADECIMIENTOS}

Se agradece a la Fundación AVINA por el financiamiento brindado al proyecto "El manejo 
como herramienta para la recuperación de cuencas". Agradecemos a todos aquellos que han colaborado en las tareas de relevamiento a campo.

\section{BIBLIOGRAFÍA}

Barberá, I., Renison, D. y Torres, R. C. (2018). Regeneración de Sebastiania commersoniana (Euphorbiaceae) en relación al ganado y la distancia al bosque en las sierras del centro de Argentina. Boletín de la Sociedad Argentina de Botánica, 53(3), 405-420. http://dx.doi.org/10.31055/1851.2372.v53. n3.21314

Bernasconi, J., Karlin, M., Accietto, R., Schneider, C., Rufini, S. y Arnulphi, S. (2015). Modelos de estados y transiciones: bases para el manejo de la vegetación en la Reserva Natural de la Defensa La Calera, Córdoba, Argentina. En E. Martinez Carretero y A. Dalmasso (Eds.), Restauración ecológica en la diagonal árida de la Argentina 2 (3-20). Mendoza, Argentina: CRICyTCONICET.

Brun, A., Campanella, O., Oggero, A. y Suárez, S. (2012). Cambios en el IVN y su relación con disturbios antrópicos en la vegetación serrana de Córdoba, Argentina. Revista de Teledetección, 37, 34-41. http:// www.aet.org.es/revistas/revista37/Revista_37_Todo. pdf\#page $=38$

Burkart, A. (1949). La posición sistemática del "chañar" y las especies del género Geoffroea (LeguminosaeDalbergieae). Darwiniana, 9 (1), 9-23. https://www. jstor.org/stable/23211719

Cabrera, A. L. (1976). Regiones fitogeográficas argentinas. En W. F. (eds), Enciclopedia Argentina de Agricultura y Jardinería, tomo II (1) (1-85). Buenos Aires, Argentina: ACME.

Cantero, J. J., Sfragulla, J. A., Núñez, C., Bonalumi, A. A., Mulko, J., Amuchastegui, A., Chiarini, F., Barboza, G. E. y Ariza Espinar, L. (2011). Flora de los afloramientos de mármoles y serpentinitas de las Sierras de Córdoba (Argentina). Kurtziana, 36(2), 11-45. http://www.scielo. org.ar/pdf/kurtz/v36n2/v36n2a03.pdf

Capó, E. A., Aguilar, R. y Renison, D. (2016). Livestock reduces juvenile tree growth of alien invasive species with a minimal effect on natives: a field experiment using exclosures. Biological Invasions, 18, 2943-2950. https://doi.org/10.1007/s10530-016-1185-3

Chazdon, R. L. (2008). Beyond deforestation: restoring forests and ecosystem services on degraded lands. Science, 320(5882), 1458-1460. https://doi. org/10.1126/science.1155365.

Di Rienzo, J., Casanoves, F., González, L., Tablada, M., Robledo C. y Balzarini, M. Infostat. (Versión 2019) [Software de cómputo] Córdoba, Argentina: Grupo
InfoStat, FCA, Universidad Nacional de Córdoba. URL: http://www.infostat.com.ar

Gaillard de Benitez, C. y Pece, M. G. (2011). Muestreo y técnicas de evaluación de vegetación y fauna. Serie Didáctica № 27. Santiago del Estero, Argentina: Facultad de Ciencias Forestales, Universidad Nacional de Santiago del Estero.

Giorgis, M. A., Cingolani, A. M. y Cabido, M. R. (2013). El efecto del fuego y las características topográficas sobre la vegetación y las propiedades del suelo en la zona de transición entre bosques y pastizales de las sierras de Córdoba, Argentina. Boletín de la Sociedad Argentina de Botánica, 48(3-4), 493-513. http://dx.doi. org/10.31055/1851.2372.v48.n3-4.7555

Giorgis, M. A., Cingolani, A. M., Chiarini, F., Chiapella, J., Barboza, G., Ariza Espinar, L., ... Cabido, M. (2011a). Composición florística del Bosque Chaqueño Serrano de la provincia de Córdoba, Argentina. Kurtziana, 36(1), 9-43. http://www.scielo.org.ar/pdf/kurtz/v36n1/ v36n1a02.pdf

Giorgis, M. A., Tecco, P. A., Cingolani, A. M., Renison, D., Marcora, P. y Paiaro, V. (2011b). Factors associated with woody alien species distribution in a newly invaded mountain system of central Argentina. Biological Invasions, 13, 1423-1434. https://doi. org/10.1007/s10530-010-9900-y

Karlin, M., Arnulphi, S., Alday, A., Bernasconi, J. y Accietto, R. (2016). Revegetación post-incendio en matorrales de Acacia spp. en las Sierras de Córdoba, Argentina Central. Oecologia Australis, 20(4), 464476. https://doi.org/10.4257/oeco.2016.2004.06

Karlin, M., Arnulphi, A., Alday, A., Bernasconi, J., Accietto, R., Denegri, A., ...Campitelli, P. (2014a). Efecto de la aplicación de compost sobre la revegetación natural de suelos afectados por incendios. NEXO Agropecuario, 2, 1-2. http://www.agro.unc.edu. ar/ secyt/webnexo/vol2-n1-2.html

Karlin, M., Bernasconi Salazar, J., Cora, A., Sánchez, S., Arnulphi, S. y Accietto, R. (2019). Cambios en el uso del suelo: capacidad de infiltración en el centro de Córdoba (Argentina). Ciencia del Suelo, 37(2), 196-208. http://www.suelos.org.ar/publicaciones/ Volumen37n2/1-\%20Pag\%20196-208\%20\%23435.pdf

Karlin, M., Bernasconi, J., Schneider, C., Rufini, S., Accietto, R., Arnulphi, S. y Cora, A. (2015). Aprovechamiento de la potencialidad silvopastoril como alternativa para el control de incendios en la Reserva Natural Militar La Calera, Córdoba (Argentina). En P.L. Peri (Ed.), III Congreso Nacional de Sistemas Silvopastoriles y VIII Congreso Internacional de Sistemas Agroforestales (pp. 636-640). Iguazú, Misiones: INTA.

Karlin, M. , Ontibero, F., Arnulphi, S. A. y Salazar, J. B. (2018). Caracterización edafológica de la Reserva Natural de la Defensa La Calera, Córdoba (Argentina). 
Multequina, 27, 5-22. https://www.redalyc.org/jatsRe po/428/42857911001/42857911001.pdf

Karlin, M. , Schneider, C., Rufini, S., Bernasconi, J., Accietto, R., Karlin, U. y Ferreyra, Y. (2014b). Caracterización florística de la Reserva Natural Militar Estancia La Calera. Nature and Conservation, 7(1), 6-18. https://doi.org/10.6008/SPC23182881.2014.001.0001

Landi, M. A. y Renison, D. (2010). Forestación con Polylepis australis en suelos erosionados de las Sierras Grandes de Córdoba: evaluación del uso de terrazas y vegetación nodriza. Ecología Austral, 20, 47-55. http://www.scielo.org.ar/pdf/ecoaus/v20n1/ v20n1a05.pdf

Naresh Kumar, M., Murthy, C. S., Sesha Sai, M. V. R. y Roy, P. S. (2009). On the use of Standardized Precipitation Index (SPI) for drought intensity assessment. Meteorological Applications, 16(3), 381-389. https:// doi.org/10.1002/met.136

Natale, E., Oggero, A. Marini, D. y Reinoso, H. (2014).
Restauración de bosque nativo en un área invadida por tamariscos Tamarix ramossisima en el sur de la provincia de Córdoba, Argentina. Ecosistemas, 23(2),130-136. https://www.revistaecosistemas.net/ index.php/ecosistemas/article/download/857/838.pdf

Torres, R., Giorgis, M., Trillo, C., Volkmann, L., Demaio, P., Heredia, J. y Renison, D. (2015). Supervivencia y crecimiento de especies con distinta estrategia de vida en plantaciones de áreas quemadas y no quemadas: un estudio de caso con dos especies leñosas en el Chaco Serrano, Argentina. Ecología Austral, 25(2), 135-143. http://ojs.ecologiaaustral.com.ar/index.php/ Ecologia_Austral/article/view/158/78

Verzino, G., Joseau, J., Díaz, M. y Dorado, M. (2004). Comportamiento inicial de especies nativas del Chaco Occidental en plantaciones en zonas de pastizales de altura de las Sierras de Córdoba, Argentina. Bosque, 25(1), 53-67. https://scielo.conicyt.cl/pdf/bosque/ v25n1/Art05.pdf 